\title{
On the Eigenvalue Distribution of Ricean MIMO Channels by Character Expansion of Groups
}

\author{
Alireza Ghaderipoor, Chintha Tellambura \\ Department of Electrical and Computer Engineering \\ University of Alberta \\ Email: \{ghaderi, chintha\}@ece.ualberta.ca
}

\begin{abstract}
Joint eigenvalue distribution of the noncentral complex Wishart matrix, i.e. $\mathrm{HH}^{*}$ where $\mathrm{H}$ is the nonzero-mean complex Gaussian random channel matrix of a multiple-input multiple-output (MIMO) system, is required for the analysis of Ricean MIMO channels from different aspects, including the average of mutual information between the transmitter and the receiver (ergodic capacity), when the channel gains are known to the receiver only. Previous works rely on the available results in mathematics for the joint eigenvalue distribution, obtained by integration over unitary matrices using classic integration methods. In this paper, we present a powerful integration method over unitary matrices which exploits the representation theory and characters of groups. The method was originally proposed for square matrices. We modify the approach from square matrices to rectangular matrices to solve a more general integral over unitary matrices and obtain the joint eigenvalue distribution of the noncentral Wishart matrix. Our result is the generalization of the previous classical integral over unitary matrices so that the result is not restricted to diagonal and/or real matrices, particularly.
\end{abstract}

\section{INTRODUCTION}

Multiple-input multiple-output (MIMO) wireless channels, which deploy antenna arrays at both the transmitter and receiver, promise high capacity and high quality wireless communication links [1], [2]. MIMO systems have been investigated in terms of the ergodic capacity [1] and the outage probability [3], by exact or asymptotic analysis [4], [5]. In the case of exact analysis, several results regarding the distribution of the channel matrix have been presented in the literature. These results indicate that in an independent and identically distributed (i.i.d.) Rayleigh fading environment, the capacity of a MIMO system with $N_{t}$ transmit antennas and $N_{r}$ receive antennas scales almost linearly with $\min \left(N_{t}, N_{r}\right)$ in the high signal-to-noise ratio (SNR) regime [1].

The most common method used in the literature for analyzing the capacity of MIMO systems is to calculate the moment generating function (MGF) of the mutual information between the transmitter and the receiver. This is done for various statistical channel models. The differentiation of the MGF yields the first moment, which will be the capacity. Moreover, the probability of outage can be derived through a simple numerical integral [6]. The outage mutual information for Gaussian uncorrelated channels by using the MGF is presented in [3], and the mutual information of MIMO systems can be found in [7] when the channel is Ricean and in [8][10] when the channel is correlated. These works are based on the available results in the theory of Wishart random matrices [11], i.e. $\mathbf{H H}^{*}$ where $\mathbf{H}$ is the complex Gaussian random channel matrix of a MIMO system. In particular, in [7], the authors use the joint probability density function (pdf) of the eigenvalues of the non-central Wishart matrix [12] to calculate the MGF of the mutual information. The used pdf [12] is a hypergeometric function with two matrix arguments.

Recently, the character expansion method, introduced by Balantekin [13], has been exploited in [14] to calculate the joint pdf of the eigenvalues of Wishart matrices. In [13], the character expansions are used for integration over unitary matrices where the coefficient matrices are nonzero-determinant square matrices. However, when the channel matrix is not a square matrix, the integrations are over unitary matrices with rectangular coefficient matrices. To handle the integrations with non-square coefficient matrices, a framework is proposed in [14]. We believe that the integration steps presented in [14] are inaccurate, which result in incorrect joint eigenvalue distributions. In this paper, after a brief introduction to the character expansion of groups, we present a modified framework to use the character expansions for integrations over unitary matrices. We show that by using the modified framework, one can easily calculate the integrations involving general rectangular complex coefficient matrices appearing in the integrand. Our result is a generalization of previous classical integral over unitary matrices so that the result is not restricted to diagonal and/or real matrices. The joint pdf of the eigenvalues of the Ricean MIMO channel matrix is a special case of our general integration.

\section{SYSTEM MODEL AND CAPACITY}

Consider a narrow-band, flat-fading communication system with $N_{t}$ transmit and $N_{r}$ receive antennas $\left(\operatorname{MIMO}\left(N_{t}, N_{r}\right)\right.$ ). The linear transformation between the transmit and receive antennas can be modeled as

$$
\mathbf{x}=\sqrt{\rho} \mathbf{H s}+\mathbf{v}
$$

where $\mathbf{x} \in \mathcal{C}^{N_{r}}$ is the complex received vector, $\mathbf{s} \in \mathcal{C}^{N_{t}}$ is the transmitted vector, $\mathbf{v} \in \mathcal{C}^{N_{r}}$ is the additive noise and $\mathbf{H} \in \mathcal{C}^{N_{r} \times N_{t}}$ is the channel matrix. To obtain the capacity, we assume the entries of both vectors $\mathbf{s}$ and $\mathbf{v}$ are independent and identically distributed (i.i.d.) complex Gaussian random variables with zero mean and variance one, $\mathcal{C N}(0,1)$. Thus, $\mathrm{E}\left\{\mathbf{s s}^{*}\right\}=\mathbf{I}$ where $\mathrm{E}\{\cdot\}$ and $(\cdot)^{*}$ denote the expectation and 
Hermitian (transpose conjugate) and $\mathbf{I}$ is the identity matrix, and $\rho$ will be the average transmitted power at each signaling interval from each antenna. To model a Ricean MIMO channel, we assume $\mathbf{H}=\mathbf{H}_{\mu}+\mathbf{H}_{w}$ where $\mathbf{H}_{\mu}$ represents the mean of $\mathbf{H}$ and is constant, and entries of $\mathbf{H}_{w}$ are i.i.d. $\mathcal{C N}(0,1)$. Note that since there is no constraint on the elements of $\mathbf{H}_{\mu}$, the entries of $\mathbf{H}$ are independent but not identically distributed. Clearly, the i.i.d. case is a special case of the above assumption.

Assuming that the channel matrix in known to the receiver only, the mutual information between the transmitter and the receiver is obtain by

$$
\mathcal{I}=\log \left(\operatorname{det}\left[\mathbf{I}+\rho \mathbf{H H}^{*}\right]\right) \quad \text { nats } / \mathrm{s} / \mathrm{Hz},
$$

where $\log (\cdot)$ denotes the natural logarithm. By defining the MGF of $\mathcal{I}$ as

$$
g(z)=\mathrm{E}_{\mathbf{H}}\left\{e^{z \mathcal{I}}\right\}=\mathrm{E}_{\mathbf{H}}\left\{\operatorname{det}\left[\mathbf{I}+\rho \mathbf{H H}^{*}\right]^{z}\right\},
$$

the capacity of the system is obtained by direct differentiation:

$$
C=\mathrm{E}_{\mathbf{H}}\{\mathcal{I}\}=g^{\prime}(0) .
$$

From (3), it is clear that the generating function can be written very simply in terms of the eigenvalues $\lambda_{i}$ of the matrix $\mathbf{H H}^{*}$ as

$$
\begin{aligned}
g(z) & =\mathrm{E}_{\boldsymbol{\lambda}}\left\{\prod_{i=1}^{M}\left(1+\rho \lambda_{i}\right)^{z}\right\} \\
& =\prod_{i=1}^{M} \int_{0}^{\infty} d \lambda_{i}\left(1+\rho \lambda_{i}\right)^{z} P\left(\left\{\lambda_{i}\right\}\right)
\end{aligned}
$$

where we define $M=\min \left\{N_{t}, N_{r}\right\}$ and $N=\max \left\{N_{t}, N_{r}\right\}$, and $P\left(\left\{\lambda_{i}\right\}\right)$ is the joint pdf of the eigenvalues of $\mathbf{H H}^{*}$. Assuming the singular value decomposition of $\mathbf{H}$ as $\mathbf{H}=$ $\mathbf{U} \boldsymbol{\Sigma} \mathbf{V}^{*}$ where $\mathbf{U} \in \mathcal{U}\left(N_{t}\right)$ (the group of unitary matrices with dimension $\left.N_{t}\right), \mathbf{V} \in \mathcal{U}\left(N_{r}\right)$ and $\boldsymbol{\Sigma}=\operatorname{diag}\left(\left\{\sqrt{\lambda_{i}}\right\}\right) \in$ $\mathcal{R}_{+}^{N_{r} \times N_{t}}$, it is shown [15] that

$$
\begin{aligned}
P\left(\left\{\lambda_{i}\right\}\right)= & K_{M, N} \Delta(\boldsymbol{\lambda})^{2} \prod_{i=1}^{M} \lambda_{i}^{N-M} \\
& \times \int D \mathbf{V} \int D \mathbf{U} p\left(\mathbf{H}=\mathbf{U} \boldsymbol{\Sigma} \mathbf{V}^{*}\right)
\end{aligned}
$$

where $K_{M, N}^{-1}=\prod_{j=1}^{M} j !(N-M+j-1) !$,

$$
\Delta(\boldsymbol{\lambda})=\operatorname{det}\left[\lambda_{i}^{j-1}\right]=\prod_{i>j}\left(\lambda_{i}-\lambda_{j}\right)
$$

is the Vandermonde determinant of vector $\boldsymbol{\lambda}(\operatorname{det}[f(i, j)]$ denotes the determinant of a matrix with the $(i, j)$ th element given by $f(i, j)), D \mathbf{U}$ denotes the Haar measure of $\mathcal{U}\left(N_{t}\right)$ [11] and $p(\mathbf{H})$ is the pdf of $\mathbf{H}$ defined as

$$
p(\mathbf{H})=\operatorname{etr}\left\{-\left(\mathbf{H}-\mathbf{H}_{\mu}\right)\left(\mathbf{H}-\mathbf{H}_{\mu}\right)^{*}\right\}
$$

where $\operatorname{etr}\{\mathbf{A}\}=\exp (\operatorname{tr}\{\mathbf{A}\})$.
Hence, for the Ricean MIMO channel

$$
\begin{array}{r}
P\left(\left\{\lambda_{i}\right\}\right)=K_{M, N} \Delta(\boldsymbol{\lambda})^{2} \prod_{j=1}^{Q} e^{-\mu_{j}} \prod_{i=1}^{M}\left[e^{-\lambda_{i}} \lambda_{i}^{N-M}\right] \times \\
\int D \mathbf{V} \int D \mathbf{U} \operatorname{etr}\left\{\mathbf{U} \boldsymbol{\Sigma} \mathbf{V}^{*} \mathbf{H}_{\mu}^{*}+\mathbf{H}_{\mu} \mathbf{V} \boldsymbol{\Sigma}^{*} \mathbf{U}^{*}\right\}
\end{array}
$$

where $\mu_{j}$ 's, $j=1, \ldots, Q$ are the $Q$ nonzero eigenvalues of $\mathbf{H}_{\mu}^{*} \mathbf{H}_{\mu}$. To find $P\left(\left\{\lambda_{i}\right\}\right)$, we define the following integral which has more general form than (8):

$$
\mathcal{J}=\int D \mathbf{V} \int D \mathbf{U} \operatorname{etr}\left\{\mathbf{U A V}^{*} \mathbf{B}+\mathbf{C V D U}^{*}\right\}
$$

where $\mathbf{U} \in \mathcal{U}(N), \mathbf{V} \in \mathcal{U}(M)$, and $\mathbf{A}, \mathbf{C} \in \mathcal{C}^{N \times M}$ and $\mathbf{B}, \mathbf{D} \in \mathcal{C}^{M \times N}$ are general rectangular complex coefficient matrices. Without loss of generality, we assume $N \geqslant M$ and that all coefficient matrices have full rank of $M$. To our best knowledge, this integral was previously known only for the case of $\mathbf{D}=\mathbf{A}^{*}$ and $\mathbf{B}=\mathbf{C}^{*}$ [12], [16] by using traditional mathematical methods, and in [14] by using the character expansion of groups. We show that the attempt in [14] is not correct and solve (9) in its general form, which is our main contribution in this paper.

\section{Character EXPANSION OF GROUPS}

The group of unitary matrices $\mathcal{U}(N)$ is a subgroup of the group of complex invertible matrices with dimension $N$ denoted by $G l(N)$. A $d$-dimensional representation of the group $G l(N)$ is a homomorphism from $G l(N)$ into the $G l(d)$. The irreducible representations of $G l(N)$ can be labeled by the $N$-dimensional ordered sets as $\mathbf{r}_{N}=\left\{r_{1}, r_{2}, \ldots, r_{N}\right\}$ where $r_{1} \geqslant r_{2} \geqslant \cdots \geqslant r_{N} \geqslant 0$ are integers. The dimension $d_{\mathbf{r}_{N}}$ of the irreducible representation $\mathbf{r}_{N}$ is given by [17]

$$
d_{\mathbf{r}_{N}}=\left[\prod_{i=1}^{N} \frac{\left(r_{i}+N-i\right) !}{(N-i) !}\right] \operatorname{det}\left[\frac{1}{\left(r_{i}-i+j\right) !}\right]
$$

where the matrix elements inside the determinant with $r_{i}$ $i+j<0$ are zero.

The character of a group element $\mathbf{X} \in G l(N)$ in its representation $\mathbf{r}_{N}$ is defined by Weyl's character formula as [18]

$$
\chi_{\mathbf{r}_{N}}(\mathbf{X})=\operatorname{tr}\left\{\mathbf{X}^{\left(\mathbf{r}_{N}\right)}\right\}=\frac{\operatorname{det}\left[x_{i}^{r_{j}+N-j}\right]}{\Delta\left(x_{1}, \ldots, x_{N}\right)}
$$

where $\mathbf{X}^{\left(\mathbf{r}_{N}\right)}$ denotes the $d_{\mathbf{r}_{N}}$ dimensional representation matrix of $\mathbf{X}$ and $\left\{x_{1}, \ldots, x_{N}\right\}$ are the eigenvalues of $\mathbf{X}$. In this case, the following equation holds for $\mathbf{X}$ [13]:

$$
\operatorname{etr}\{\mathbf{X}\}=\sum_{\mathbf{r}_{N}} \alpha_{\mathbf{r}_{N}} \chi_{\mathbf{r}_{N}}(\mathbf{X})
$$

where the summation is over all irreducible representations of $G l(N)$ and the expansion factor $\alpha_{\mathbf{r}_{N}}$ is defined as

$$
\alpha_{\mathbf{r}_{N}}=\operatorname{det}\left[\frac{1}{\left(r_{i}-i+j\right) !}\right]=\left[\prod_{i=1}^{N} \frac{(N-i) !}{\left(r_{i}+N-i\right) !}\right] d_{\mathbf{r}_{N}}
$$


Lemma 1: The orthogonality relation between unitary matrix group elements implies that [19]

$$
\int D \mathbf{U} U_{i j}^{\left(\mathbf{r}_{N}\right)} U_{k l}^{\left(\mathbf{r}_{N}^{\prime}\right) *}=\frac{1}{d_{\mathbf{r}_{N}}} \delta_{\mathbf{r}_{N} \mathbf{r}_{N}^{\prime}} \delta_{i k} \delta_{j l}
$$

where $U_{i j}^{\left(\mathbf{r}_{N}\right)}$ denotes the $(i, j)$ th element of the representation matrix of $\mathbf{U}$ and $d_{\mathbf{r}_{N}}$ is the dimension of the representation.

Lemma 2: Cauchy-Binet formula [20]: Given vectors $\mathbf{x}$ and $\mathbf{y}$ with dimension $N$ and a power series expansion $f(z)=$ $\sum_{i=0}^{\infty} a(i) z^{i}$ convergent for $|z|<\xi$, then if $\left|x_{i} y_{j}\right|<\xi$ for all $1 \leqslant i, j \leqslant N$, one can write

$$
\begin{aligned}
& \operatorname{det}\left[f\left(x_{i} y_{j}\right)\right]= \\
& \sum_{r_{1}>r_{2}>\cdots>r_{N} \geqslant 0} \operatorname{det}\left[x_{i}^{r_{j}}\right] \operatorname{det}\left[y_{i}^{r_{j}}\right] \prod_{i=1}^{N} a\left(r_{i}\right)
\end{aligned}
$$

Proposition 1: Assuming A, $\mathbf{B} \in G l(N), \mathbf{U} \in \mathcal{U}(N)$ and $\mathbf{r}_{N}$ and $\mathbf{r}_{N}^{\prime}$ two representations of $G l(N)$, then

$$
\int D \mathbf{U} \chi_{\mathbf{r}_{N}}(\mathbf{A U}) \chi_{\mathbf{r}_{N}^{\prime}}\left(\mathbf{B} \mathbf{U}^{*}\right)=\frac{1}{d_{\mathbf{r}_{N}}} \chi_{\mathbf{r}_{N}}(\mathbf{A B}) \delta_{\mathbf{r}_{N} \mathbf{r}_{N}^{\prime}} .
$$

Proof: From (11), we have $\chi_{\mathbf{r}_{N}}(\mathbf{A U})=\operatorname{tr}\left\{(\mathbf{A U})^{\left(\mathbf{r}_{N}\right)}\right\}$. Since a representation is a homomorphism, i.e. $(\mathbf{A U})^{\left(\mathbf{r}_{N}\right)}=$ $\mathbf{A}^{\left(\mathbf{r}_{N}\right)} \mathbf{U}^{\left(\mathbf{r}_{N}\right)}$, we obtain

$$
\chi_{\mathbf{r}_{N}}(\mathbf{A U})=\operatorname{tr}\left\{\mathbf{A}^{\left(\mathbf{r}_{N}\right)} \mathbf{U}^{\left(\mathbf{r}_{N}\right)}\right\}=\sum_{k_{2}=1}^{N} \sum_{k_{1}=1}^{N} A_{k_{2} k_{1}}^{\left(\mathbf{r}_{N}\right)} U_{k_{1} k_{2}}^{\left(\mathbf{r}_{N}\right)}
$$

Therefore, we have

$$
\begin{aligned}
& \int D \mathbf{U}_{\mathbf{r}_{N}}(\mathbf{A U}) \chi_{\mathbf{r}_{N}^{\prime}}\left(\mathbf{B U}^{*}\right) \\
& =\sum_{k_{4}=1}^{N} \sum_{k_{3}=1}^{N} \sum_{k_{2}=1}^{N} \sum_{k_{1}=1}^{N} A_{k_{2} k_{1}}^{\left(\mathbf{r}_{N}\right)} B_{k_{4} k_{3}}^{\left(\mathbf{r}_{N}^{\prime}\right)} \int D \mathbf{U} U_{k_{1} k_{2}}^{\left(\mathbf{r}_{N}\right)} U_{k_{4} k_{3}}^{\left(\mathbf{r}_{N}^{\prime}\right) *} \\
& =\frac{\delta_{\mathbf{r}_{N}} \mathbf{r}_{N}^{\prime}}{d_{\mathbf{r}_{N}}} \sum_{k_{2}=1}^{N} \sum_{k_{1}=1}^{N} A_{k_{2} k_{1}}^{\left(\mathbf{r}_{N}\right)} B_{k_{1} k_{2}}^{\left(\mathbf{r}_{N}\right)}=\frac{1}{d_{\mathbf{r}_{N}}} \chi_{\mathbf{r}_{N}}(\mathbf{A B}) \delta_{\mathbf{r}_{N} \mathbf{r}_{N}^{\prime}}
\end{aligned}
$$

where the second equality comes from Lemma 1 .

Proposition 2: Assuming $\mathbf{A}, \mathbf{B} \in G l(N), \mathbf{U} \in \mathcal{U}(N)$ and $\mathbf{r}_{N}$ a representation of $G l(N)$, then

$$
\int D \mathbf{U} \chi_{\mathbf{r}_{N}}\left(\mathbf{A} \mathbf{U B U} \mathbf{U}^{*}\right)=\frac{1}{d_{\mathbf{r}_{N}}} \chi_{\mathbf{r}_{N}}(\mathbf{A}) \chi_{\mathbf{r}_{N}}(\mathbf{B}) .
$$

Proof: From (11), we have

$$
\begin{aligned}
\chi_{\mathbf{r}_{N}}\left(\mathbf{A U B U}^{*}\right)=\operatorname{tr}\left\{\mathbf{A}^{\left(\mathbf{r}_{N}\right)} \mathbf{U}^{\left(\mathbf{r}_{N}\right)} \mathbf{B}^{\left(\mathbf{r}_{N}\right)} \mathbf{U}^{\left(\mathbf{r}_{N}\right) *}\right\} \\
=\sum_{k_{4}=1}^{N} \sum_{k_{3}=1}^{N} \sum_{k_{2}=1}^{N} \sum_{k_{1}=1}^{N} A_{k_{4} k_{3}}^{\left(\mathbf{r}_{N}\right)} U_{k_{3} k_{2}}^{\left(\mathbf{r}_{N}\right)} B_{k_{2} k_{1}}^{\left(\mathbf{r}_{N}\right)} U_{k_{4} k_{1}}^{\left(\mathbf{r}_{N}\right) *}
\end{aligned}
$$

Therefore,

$$
\begin{aligned}
& \int D \mathbf{U} \chi_{\mathbf{r}_{N}}\left(\mathbf{A} \mathbf{U B U} \mathbf{U}^{*}\right) \\
& =\sum_{k_{4}=1}^{N} \sum_{k_{3}=1}^{N} \sum_{k_{2}=1}^{N} \sum_{k_{1}=1}^{N} A_{k_{4} k_{3}}^{\left(\mathbf{r}_{N}\right)} B_{k_{2} k_{1}}^{\left(\mathbf{r}_{N}\right)} \int D \mathbf{U} U_{k_{3} k_{2}}^{\left(\mathbf{r}_{N}\right)} U_{k_{4} k_{1}}^{\left(\mathbf{r}_{N}\right) *} \\
& =\frac{1}{d_{\mathbf{r}_{N}}} \sum_{k_{3}=1}^{N} \sum_{k_{1}=1}^{N} A_{k_{3} k_{3}}^{\left(\mathbf{r}_{N}\right)} B_{k_{1} k_{1}}^{\left(\mathbf{r}_{N}\right)}=\frac{1}{d_{\mathbf{r}_{N}}} \chi_{\mathbf{r}_{N}}(\mathbf{A}) \chi_{\mathbf{r}_{N}}(\mathbf{B})
\end{aligned}
$$

\section{Calculation of $\mathcal{J}$}

Recall that we assumed $N \geqslant M$ in (9). Considering the rank of matrices in (9), if we define $\mathbf{E}=\mathbf{A V}^{*} \mathbf{B}$ and $\mathbf{F}=\mathbf{C V D}$, both $N$-dimensional matrices $\mathbf{E}$ and $\mathbf{F}$ are of the rank $M$ and are not a member of $G l(N)$. Thus, we need more assumptions to use the character expansions. We assume that both $\mathbf{E}$ and $\mathbf{F}$ have a full rank of $N$, to use $N$-dimensional representations and the character expansions and obtain:

$$
\begin{aligned}
\mathcal{J}_{1} & =\int D \mathbf{V} \int D \mathbf{U} \operatorname{etr}\{\mathbf{E U}\} \operatorname{etr}\left\{\mathbf{F} \mathbf{U}^{*}\right\} \\
& =\sum_{\mathbf{r}_{N}} \sum_{\mathbf{r}_{N}^{\prime}} \alpha_{\mathbf{r}_{N}} \alpha_{\mathbf{r}_{N}^{\prime}} \int D \mathbf{V} \int D \mathbf{U} \chi_{\mathbf{r}_{N}}(\mathbf{E U}) \chi_{\mathbf{r}_{N}^{\prime}}\left(\mathbf{F} \mathbf{U}^{*}\right) \\
& =\sum_{\mathbf{r}_{N}} \frac{\alpha_{\mathbf{r}_{N}}^{2}}{d_{\mathbf{r}_{N}}} \int D \mathbf{V} \chi_{\mathbf{r}_{N}}(\mathbf{E F}) \\
& =\sum_{\mathbf{r}_{N}} \frac{\alpha_{\mathbf{r}_{N}}^{2}}{d_{\mathbf{r}_{N}}} \int D \mathbf{V} \chi_{\mathbf{r}_{N}}\left(\mathbf{A} \mathbf{V}^{*} \mathbf{B C V D}\right)
\end{aligned}
$$

where the second equality comes from (12) and the third equality is obtained by using the Proposition 1 . Note that the above assumptions allowed us to take the integration over $\mathcal{U}(N)$. Clearly, we must introduce the following limits to make sure that the above assumptions hold for $\mathcal{J}$ :

$$
\mathcal{J}=\lim _{\left\{\eta_{1}, \ldots, \eta_{N-M}\right\} \rightarrow 0} \lim _{\left\{r_{M+1}, \ldots, r_{N}\right\} \rightarrow 0} \mathcal{J}_{1}
$$

where the first limit guarantees that the matrices $\mathbf{E}$ and $\mathbf{F}$ or equivalently the matrix $\mathbf{A V}^{*} \mathbf{B C V D}$ have only $M$ nonzero eigenvalues; and the second limit is because we used $N$ dimensional representations to be able to integrate over $\mathcal{U}(N)$ while we were allowed to use $M$-dimensional representations only. For instance, we could use the $M$-dimensional representations for character expansion of $\operatorname{etr}\left\{\mathbf{U} \mathbf{A V} \mathbf{V}^{*} \mathbf{B}\right\}$ in (9) by using the substitution of etr $\left\{\mathbf{U} \mathbf{A V} \mathbf{V}^{*} \mathbf{B}\right\}$ with $\operatorname{etr}\left\{\mathbf{B U} \mathbf{A} V^{*}\right\}$. Because $\operatorname{tr}\left\{\mathbf{U A V}^{*} \mathbf{B}\right\}=\operatorname{tr}\left\{\mathbf{B U A N}{ }^{*}\right\}$ [21] and despite the matrix $\mathbf{U A V}^{*} \mathbf{B}$ which is not full rank, the matrix $\mathbf{B} \mathbf{U} \mathbf{A V}^{*}$ has the full rank $M$. But in this case, we could not perform the integration over $\mathcal{U}(N)$. We emphasize that the second limit in (17) is critical. The authors in [14] does not consider this point and by adding rows and columns to matrices, they practically take both integrations over $\mathcal{U}(N)$ and thus the final result is not correct.

To calculate (17), we present the following propositions: 


\section{Proposition 3:}

$$
\begin{aligned}
\lim _{\left\{r_{M+1}, \ldots, r_{N}\right\} \rightarrow 0} & \frac{\alpha_{\mathbf{r}_{N}}}{d_{\mathbf{r}_{N}}}=\lim _{\left\{r_{M+1}, \ldots, r_{N}\right\} \rightarrow 0}\left[\prod_{i=1}^{N} \frac{(N-i) !}{\left(r_{i}+N-i\right) !}\right] \\
= & \frac{\prod_{i=1}^{N}(N-i) !}{\prod_{i=1}^{M}\left(r_{i}+N-i\right) ! \prod_{i=M+1}^{N}(N-i) !} \\
= & \frac{\prod_{i=N-M}^{N-1} i !}{\prod_{i=1}^{M}\left(r_{i}+N-i\right) !} .
\end{aligned}
$$

\section{Proposition 4:}

$$
\lim _{\left\{r_{M+1}, \ldots, r_{N}\right\} \rightarrow 0} \alpha_{\mathbf{r}_{N}}=\alpha_{\mathbf{r}_{M}}
$$

Proof: From the definition of $\alpha_{\mathbf{r}_{N}}$ in (13) and noting that the matrix elements inside the determinant with $r_{i}-i+j<0$ are zero, we have

$$
\begin{aligned}
\lim _{\left\{r_{M+1}, \ldots, r_{N}\right\} \rightarrow 0} & \alpha_{\mathbf{r}_{N}}=\lim _{\left\{r_{M+1}, \ldots, r_{N}\right\} \rightarrow 0} \operatorname{det}\left[\frac{1}{\left(r_{i}-i+j\right) !}\right] \\
& =\operatorname{det}\left[\begin{array}{cc}
\mathbf{E}_{M \times M} & \mathbf{F}_{M \times(N-M)} \\
\mathbf{0}_{(N-M) \times M} & \mathbf{R}_{(N-M) \times(N-M)}
\end{array}\right] \\
& =\operatorname{det}[\mathbf{E}] \operatorname{det}[\mathbf{R}]
\end{aligned}
$$

where $E_{i j}=\left[\left(r_{i}-i+j\right) !\right]^{-1}$ and

$$
\mathbf{R}=\left[\begin{array}{cccc}
\frac{1}{0 !} & \frac{1}{1 !} & \cdots & \frac{1}{(N-M-1) !} \\
0 & \frac{1}{0 !} & \cdots & \frac{1}{(N-M-2) !} \\
\vdots & \vdots & \ddots & \vdots \\
0 & 0 & \cdots & \frac{1}{0 !}
\end{array}\right]
$$

so that $\operatorname{det}[\mathbf{R}]=1$. Thus,

$$
\lim _{\left\{r_{M+1}, \ldots, r_{N}\right\} \rightarrow 0} \alpha_{\mathbf{r}_{N}}=\operatorname{det}[\mathbf{E}]=\alpha_{\mathbf{r}_{M}}
$$

Lemma 3: If we define the following ratio

$$
R\left(x_{1}, \ldots, x_{N}\right)=\frac{\operatorname{det}\left[f_{i}\left(x_{j}\right)\right]}{\Delta\left(x_{1}, \ldots, x_{N}\right)}
$$

where $i, j=1, \ldots, N$, then

$$
\begin{aligned}
& \lim _{\left\{x_{1}, \ldots, x_{p}\right\} \rightarrow 0} R\left(x_{1}, \ldots, x_{N}\right) \\
& \quad=\frac{\operatorname{det}[\mathbf{Z}]}{\Delta\left(x_{p+1}, \ldots, x_{N}\right) \prod_{i=p+1}^{N} x_{i}^{p} \prod_{j=1}^{p-1} j !}
\end{aligned}
$$

where

$$
\mathbf{Z}=\left[f_{i}(0), f_{i}^{(1)}(0), \ldots, f_{i}^{(p-1)}(0), f_{i}\left(x_{p+1}\right), \ldots, f_{i}\left(x_{N}\right)\right]
$$

where $i=1, \ldots, N$ generates all rows of $\mathbf{Z}$ and $f^{(k)}$ denotes the $k$ th derivative of the function $f$. (See Lemma 6 in [14] for proof.)

Proposition 5: Assuming $\mathbf{A}_{N \times M}, \mathbf{G}_{M \times M}$ and $\mathbf{D}_{M \times N}$ are of the rank $M(N \geqslant M)$, and $x_{i}$ 's $i=1, \ldots, N$ are the eigenvalues of the matrix AGD, then

$\lim _{\left\{x_{1}, \ldots, x_{N-M}\right\} \rightarrow 0} \lim _{\left\{r_{M+1}, \ldots, r_{N}\right\} \rightarrow 0} \chi_{\mathbf{r}_{N}}($ AGD $)=\chi_{\mathbf{r}_{M}}(\mathbf{D A G})$ where $\left\{x_{1}, \ldots, x_{N-M}\right\}$ represent the $N-M$ zero eigenvalues of AGD.

Proof: From (11) and noting that $\operatorname{det}\left[x_{i}^{r_{j}+N-j}\right]=$ $\operatorname{det}\left[x_{j}^{r_{i}+N-i}\right]$, we have

$$
\begin{aligned}
\lim _{\left\{r_{M+1}, \ldots, r_{N}\right\} \rightarrow 0} \chi_{\mathbf{r}_{N}}(\mathbf{A G D}) & =\lim _{\left\{r_{M+1}, \ldots, r_{N}\right\} \rightarrow 0} \frac{\operatorname{det}\left[x_{i}^{r_{j}+N-j}\right]}{\Delta\left(x_{1}, \ldots, x_{N}\right)} \\
& =\frac{\operatorname{det}[\mathbf{X}]}{\Delta\left(x_{1}, \ldots, x_{N}\right)}
\end{aligned}
$$

where

$$
X_{i j}= \begin{cases}x_{j}^{r_{i}+N-i}, & i \leqslant M \\ x_{j}^{N-i}, & i>M\end{cases}
$$

Now by taking $f_{i}\left(x_{j}\right)=X_{i j}$ as defined above and applying Lemma 3 , it is easy to see that

$$
\begin{aligned}
& \lim _{\left\{x_{1}, \ldots, x_{N-M}\right\} \rightarrow 0} \frac{\operatorname{det}[\mathbf{X}]}{\Delta\left(x_{1}, \ldots, x_{N}\right)} \\
& =\frac{\operatorname{det}\left[\begin{array}{cc}
\mathbf{0}_{M \times(N-M)} & \mathbf{Q}_{M \times M} \\
\mathbf{P}_{(N-M) \times(N-M)} & \mathbf{T}_{(N-M) \times M}
\end{array}\right]}{\Delta\left(x_{N-M+1}, \ldots, x_{N}\right) \prod_{i=N-M+1}^{N} x_{i}^{N-M} \prod_{j=1}^{N-M-1} j !}
\end{aligned}
$$

where

$$
\mathbf{P}=\left[\begin{array}{ccccc}
0 & 0 & 0 & \cdots & (N-M-1) ! \\
\vdots & \vdots & \vdots & \ddots & \vdots \\
0 & 0 & 2 ! & \cdots & 0 \\
0 & 1 ! & 0 & \cdots & 0 \\
0 ! & 0 & 0 & \cdots & 0
\end{array}\right]
$$

and

$$
\mathbf{Q}=\left[\begin{array}{ccc}
x_{N-M+1}^{r_{1}+N-1} & \cdots & x_{N}^{r_{1}+N-1} \\
\vdots & \ddots & \vdots \\
x_{N-M+1}^{r_{M}+N-M} & \cdots & x_{N}^{r_{M}+N-M}
\end{array}\right]
$$

By column factoring of $\mathbf{Q}$, we obtain

$$
\begin{aligned}
\operatorname{det}\left[\begin{array}{ll}
\mathbf{0} & \mathbf{Q} \\
\mathbf{P} & \mathbf{T}
\end{array}\right] & =\prod_{j=0}^{N-M-1} j ! \operatorname{det}[\mathbf{Q}] \\
& =\prod_{j=1}^{N-M-1} j ! \prod_{i=N-M+1}^{N} x_{i}^{N-M} \operatorname{det}\left[x_{N-M+j}^{r_{i}+M-i}\right]
\end{aligned}
$$

Therefore,

$$
\begin{aligned}
& \lim _{\left\{x_{1}, \ldots, x_{N-M}\right\} \rightarrow 0} \lim _{\left\{r_{M+1}, \ldots, r_{N}\right\} \rightarrow 0} \chi_{\mathbf{r}_{N}}(\mathbf{A G D}) \\
&=\frac{\operatorname{det}\left[x_{N-M+j}^{r_{i}+M-i}\right]}{\Delta\left(x_{N-M+1}, \ldots, x_{N}\right)}=\chi_{\mathbf{r}_{M}}(\mathbf{D A G})
\end{aligned}
$$

where the last equality comes from the fact that the nonzero eigenvalues of the matrices AGD and DAG are equal [21]. 
To continue the calculation of $\mathcal{J}$, from (17) we have

$$
\begin{gathered}
\mathcal{J}=\int D \mathbf{V}_{\left\{\eta_{1}, \ldots, \eta_{N-M}\right\} \rightarrow 0} \lim _{\left\{r_{M+1}, \ldots, r_{N}\right\} \rightarrow 0} \sum_{\mathbf{r}_{N}}\left[\frac{\alpha_{\mathbf{r}_{N}}}{d_{\mathbf{r}_{N}}}\right] \alpha_{\mathbf{r}_{N}} \chi_{\mathbf{r}_{N}}\left(\mathbf{A V}^{*} \mathbf{B C V D}\right)
\end{gathered}
$$

By applying Propositions 3, 4 and 5, we have

$$
\begin{aligned}
\mathcal{J} & =\sum_{\mathbf{r}_{M}} \frac{\prod_{i=N-M}^{N-1} i !}{\prod_{i=1}^{M}\left(N-i+r_{i}\right) !} \alpha_{\mathbf{r}_{M}} \int D \mathbf{V} \chi_{\mathbf{r}_{M}}\left(\mathbf{D A} \mathbf{A V}^{*} \mathbf{B C V}\right) \\
& =\sum_{\mathbf{r}_{M}} \frac{\prod_{i=N-M}^{N-1} i !}{\prod_{i=1}^{M}\left(N-i+r_{i}\right) !} \frac{\alpha_{\mathbf{r}_{M}}}{d_{\mathbf{r}_{M}}} \chi_{\mathbf{r}_{M}}(\mathbf{D A}) \chi_{\mathbf{r}_{M}}(\mathbf{B C})
\end{aligned}
$$

where the second equality comes from Proposition 2. By applying the Weyl's formula (11) and (13), we obtain

$$
\begin{aligned}
\mathcal{J}= & {\left[\prod_{n=N-M}^{N-1} n !\right]\left[\prod_{m=1}^{M-1} m !\right] \frac{1}{\Delta(\mathbf{x}) \Delta(\mathbf{y})} \times } \\
& \sum_{\mathbf{r}_{M}} \operatorname{det}\left[x_{i}^{k_{j}}\right] \operatorname{det}\left[y_{i}^{k_{j}}\right] \prod_{i=1}^{M} \frac{1}{k_{i} !\left(k_{i}+N-M\right) !}
\end{aligned}
$$

where $k_{i} \triangleq M-i+r_{i}$ and $M$-dimensional vectors $\mathbf{x}$ and $\mathbf{y}$ are the eigenvalues of matrices $\mathbf{D A}$ and $\mathbf{B C}$, respectively.

Considering the power series expansion of the modified Bessel function as

$$
\frac{I_{z}(2 x)}{x^{z}}=\sum_{k=0}^{\infty} \frac{x^{2 k}}{k !(k+z) !}
$$

and by applying the Cauchy-Binet formula (15), we obtain

$$
\begin{aligned}
\mathcal{J} & =\left[\prod_{n=N-M}^{N-1} n !\right]\left[\prod_{m=1}^{M-1} m !\right] \frac{\operatorname{det}\left[\left(x_{i} y_{j}\right)^{\frac{M-N}{2}} I_{N-M}\left(2 \sqrt{x_{i} y_{j}}\right)\right]}{\Delta(\mathbf{x}) \Delta(\mathbf{y})} \\
& =\left[\prod_{n=N-M}^{N-1} n !\right]\left[\prod_{m=1}^{M-1} m !\right] \frac{\operatorname{det}\left[I_{N-M}\left(2 \sqrt{x_{i} y_{j}}\right)\right]}{\Delta(\mathbf{x}) \Delta(\mathbf{y}) \prod_{i=1}^{M}\left(x_{i} y_{i}\right)^{\frac{N-M}{2}}} .
\end{aligned}
$$

\section{Joint PDF OF EIGENVALUES OF $\mathbf{H}$}

If we assume all $M$ eigenvalues of $\mathbf{H}_{\mu}^{*} \mathbf{H}_{\mu}$ are nonzero, we can directly apply (21) to (8) to obtain

$$
\begin{aligned}
P\left(\left\{\lambda_{i}\right\}\right) & =K_{M, N}\left[\prod_{n=N-M}^{N-1} n !\right]\left[\prod_{m=1}^{M-1} m !\right] \frac{\Delta(\boldsymbol{\lambda})}{\Delta(\boldsymbol{\mu})} \prod_{j=1}^{M} e^{-\lambda_{j}-\mu_{j}} \\
& \times \prod_{i=1}^{M}\left[\sqrt{\frac{\lambda_{i}}{\mu_{i}}}\right]^{N-M} \operatorname{det}\left[I_{N-M}\left(2 \sqrt{\lambda_{i} \mu_{j}}\right)\right]
\end{aligned}
$$

This result is consistent with the results in [12] and [16] obtained by using classic mathematical methods. Although the authors in [14] have applied the character expansion of groups to find $P\left(\left\{\lambda_{i}\right\}\right)$ for Ricean MIMO channels, the final result is in the form of $I_{0}\left(2 \sqrt{\lambda_{i} \mu_{j}}\right)$ and therefore is incorrect.
For the case that some of eigenvalues of $\mathbf{H}_{\mu}^{*} \mathbf{H}_{\mu}$ are equal to zero, one can use the Lemma 3 to obtain $P\left(\left\{\lambda_{i}\right\}\right)$. For instance, in the case of i.i.d. $\mathbf{H}$ where all entries of $\mathbf{H}_{\mu}$ are equal, $\mathbf{H}_{\mu}^{*} \mathbf{H}_{\mu}$ has only one nonzero eigenvalue. Due to the page limits, we omit the i.i.d part, which will appear in the journal version of the paper.

\section{ACKNOWLEDGEMENTS}

The authors would like to thank iCORE Wireless Communications Laboratory and Alberta Ingenuity Fund for supporting our research.

\section{REFERENCES}

[1] E. Telatar, "Capacity of multiantenna Gaussian channels," AT\&T Bell Laboratories, Tech. Memo., 1995.

[2] G. J. Foschini and M. J. Gans, "On limits of wireless communications in a fading environment when using multiple antennas," Wireless Personal Commun., vol. 6, pp. 311-335, March 1998.

[3] Z. Wang and G. B. Giannakis, "Outage mutual information of spacetime MIMO channels," IEEE Trans. Inform. Theory, vol. 50, no. 4, pp. 657-662, Apr. 2004.

[4] J. Dumont, P. Loubaton, S. Lasaulce, and M. Debbah, "On the asymptotic performance of MIMO correlated Rician channels," in Proc. IEEE Int. Conf. Acoust., Speech, Signal Process. (ICASSP), Philadelphia, PA, 2005, pp. 22-25.

[5] E. Biglieri, G. Taricco, and A. Tulino, "Performance of space-time codes for a large number of antennas," IEEE Trans. Inform. Theory, vol. 48, no. 7, pp. 1794-1803, July 2002.

[6] L. H. Ozarow, S. Shamai, and A. D. Wyner, "Information theoretic considerations for cellular mobile radio," IEEE Trans. Veh. Technol., vol. 43, no. 2, pp. 359-378, May 1994.

[7] M. Kang and M. S. Alouini, "Capacity of MIMO Rician channels," IEEE Trans. Wireless Commun., vol. 5, no. 1, pp. 112-122, Jan. 2006.

[8] M. Chiani, M. Z. Win, and A. Zanella, "On the capacity of spatially correlated MIMO Rayleigh fading channels," IEEE Trans. Inform. Theory, vol. 49, no. 10, pp. 2363-2371, Oct. 2003.

[9] P. J. Smith, S. Roy, and M. Shafi, "Capacity of MIMO systems with semicorrelated flat fading," IEEE Trans. Inform. Theory, vol. 49, no. 10, pp. 2781-2788, Oct. 2003.

[10] M. Kiessling and J. Speidel, "Mutual information of MIMO channels in correlated Rayleigh fading environments - a general solution," in Proc. IEEE Int. Conf. on Commun. (ICC), vol. 2, June 20-24, 2004, pp. 814818.

[11] A. Edelman, "Eigenvalues and condition numbers of random matrices," Ph.D. dissertation, Department of Mathematics, MIT, May 1989.

[12] A. T. James, "Distributions of matrix variates and latent roots derived from normal samples," Ann. Math. Statist., vol. 35, no. 2, pp. 475-501, 1964.

[13] A. B. Balantekin, "Character expansions, Itzykson-Zuber integrals and the QCD partition action," Phys. Rev. D, vol. 62, no. 8, p. 5017, Oct. 2000.

[14] S. H. Simon, A. L. Moustakas, and L. Marinelli, "Capacity and character expansions: moment-generating function and other exact results for MIMO correlated channels," IEEE Trans. Inform. Theory, vol. 52, no. 12 , pp. 5336-5351, Dec. 2006.

[15] M. L. Mehta, Random Matrices, 3rd ed. NewYork: Academic Press, 2004.

[16] T. Guhr and T. Wettig, "An ItzyksonZuber-like integral and diffusion for complex ordinary and supermatrices," J. Math. Phys., vol. 37, pp. 6395-6413, Dec 1996.

[17] A. B. Balantekin and I. Bars, "Dimension and character formulas for Lie supergroups," J. Math. Phys., vol. 22, no. 6, pp. 1149-1162, 1981.

[18] H. Weyl, The Classical Groups. Princeton, NJ: Princeton University Press, 1948.

[19] S. Sternberg, Group theory and Physics. Cambridge, U.K.: Cambridge University Press, 1995.

[20] L. K. Hua, Harmonic Analysis of Functions of Several Complex Variables in the Classical Domains. Providence, RI: American Mathematical Society, 1963.

[21] R. A. Horn and C. R. Johnson, Matrix Analysis. New York: Cambridge University Press, 2001. 